\title{
INVESTIGACIONES
}

\section{Rúbrica para evaluar un diseño curricular bajo el enfoque socioformativo*}

\author{
Rubric to evaluate a curriculum \\ design under the socioformative approach
}

\begin{abstract}
Angélica Crespo-Cabuto ${ }^{a}$, Sonia Verónica Mortis-Lozoya ${ }^{a}$, Sergio de Jesús Tobón Tobón ${ }^{b}$, Sergio Raúl Herrera Meza ${ }^{b}$
\end{abstract}

\author{
${ }^{a}$ Departamento de Educación, Instituto Tecnológico de Sonora, México (ITSON). \\ angelica.crespo@itson.edu.mx, sonia.mortis@itson.edu.mx \\ ${ }^{b}$ Centro Universitario CIFE, México. \\ stobon@cife.ws, sergioherrera@cife.edu.mx
}

\begin{abstract}
RESUMEN
El enfoque socioformativo considera la instrucción de los actores del proceso educativo, favoreciendo el aprendizaje integral, atendiendo los problemas del contexto colaborativamente, con idoneidad y compromiso ético. El objetivo de esta investigación fue establecer la validez de constructo y confiabilidad de una rúbrica para evaluar la aplicación de los 12 ejes esenciales del currículo por competencias bajo el enfoque socioformativo, mediante un análisis factorial exploratorio y confirmatorio. El instrumento se aplicó a 74 responsables de gestionar el currículo a nivel licenciatura, en universidades de México y países de Latinoamérica. Los resultados indican que la rúbrica tiene solución unifactorial, explica el $63.3 \%$ de la varianza del constructo. Se concluye que la rúbrica cuenta con validez de constructo y confiabilidad $(\alpha=0.94)$, por lo que se recomienda su aplicación en universidades del país y de Latinoamérica para identificar el nivel de aplicación de los ejes esenciales del currículo.
\end{abstract}

Palabras claves: Calidad educativa, Evaluación Curricular, Validez de constructo, Confiabilidad.

\section{ABSTRACT}

The socioformative approach considers the training of the actors of the educational process, subserving the integral formation, collaboratively attending to the context's problems, with suitability and ethical commitment. The objective of this research was to establish the construct validity and reliability of a rubric to assess the application of the 12 essential axes of the competency curriculum under the socioformative approach, through exploratory and confirmatory factor analysis. The instrument was applied to 74 people responsible for managing the curriculum at a bachelor's level, in universities in Mexico and Latin American countries. The results indicate that the rubric presents unifactorial solution, explains $63.3 \%$ of the variance of the construct. It is concluded that the rubric has construct validity and reliability $(\alpha=0.94)$, so its application in universities of the country and Latin America is recommended to identify the level of application of the essential axes of the curriculum.

Key words: Educational quality, Curriculum Evaluation, Construct validity, Reliability.

\footnotetext{
* Los resultados de este estudio se obtuvieron gracias a la financiación del proyecto de investigación: "Diseño curricular y aplicación del enfoque Socioformativo en profesores de LCE”, por el Instituto Tecnológico de Sonora, a través de PFCE 2019 y el Programa de Promoción de la Investigación PROFAPI-2019-009. Para lo cual se agradece el apoyo obtenido.
} 


\section{INTRODUCCIÓN}

Las universidades se han visto impactadas por las exigencias del contexto, el cual les demanda integrar elementos que son de suma importancia para la sociedad del conocimiento. Según Tobón, Guzmán, Hernández y Cardona (2015), dichos elementos son: a) trabajo colaborativo, b) uso de las tecnologías de la información y la comunicación (TIC), c) gestión del cambio y d) gestión del conocimiento. A su vez, estos elementos inciden en los procesos educativos; los cuales requieren de la creación de programas educativos flexibles, que se adapten a los requerimientos sociales, solucionen los problemas que desfavorecen de manera directa la calidad de vida y permitan el crecimiento personal del individuo (Almonacid, Montes y Vásquez, 2009; Cevallos, 2014).

Para lograr atender los constantes desafíos, las universidades realizan procesos de diseño curricular de manera periódica con el fin de mantener la pertinencia y relevancia de los programas educativos y a su vez asegurar su calidad. Así mismo, buscan que los planes de estudio cuenten con acreditaciones por parte de organismos externos, los cuales reconocen el cumplimiento de estándares nacionales e internacionales que dan garantía a dichos procesos educativos y posiciona a la institución (Martínez, Tobón y Romero, 2017).

Ante este panorama, se ha identificado que para que el proceso de diseño curricular sea exitoso y permita que los programas educativos mantengan su reconocimiento a nivel nacional e internacional, es necesario que se consideren las exigencias sociales, con la finalidad de que las actualizaciones de la disciplina profesional sean directrices para establecer las competencias a alcanzar por los egresados y realizar mejoras en la organización del plan de estudio (Icarte y Labate, 2016; Cruz, Lemus, Ramos y Pujols, 2017).

Sin embargo, llevar a cabo estos procesos de innovación curricular en las entidades educativas de Educación Superior es un desafío constante; ya que las transformaciones sociales se viven de manera acelerada y generan urgencias que requieren cambios inmediatos en los procesos de formación. Con el fin de atender la realidad del contexto, dentro de las universidades las actualizaciones curriculares se llevan a cabo bajo plazos rigurosamente establecidos, lo cual desfavorece la participación, debate y reflexión de los expertos, y a su vez la posibilidad de ofertar programas educativos fortalecidos (Castro, Lira y Castañeda, 2017; Icarte y Labate, 2016).

Se han desarrollado diversas investigaciones orientadas al análisis de validez por medio de estudios exploratorios, así como la confiabilidad de instrumentos para evaluar el cumplimiento de los elementos que favorecen la calidad educativa. Algunos de ellos se enfocan a la práctica y habilidades del docente, como el estudio realizado por Agreda, Hinojo y Sola (2016) el cual valora la competencia digital de los profesores; así como el de Tobón, Pimienta, Herrera, Juárez y Hernández (2018), quienes validaron una rúbrica para medir las prácticas docentes efectivas.

Por otra parte, en diversos estudios se analizaron las propiedades psicométricas de instrumentos para asegurar su validez y confiabilidad, todos ellos aplicados a alumnos universitarios; tales como, los efectuados por Gutiérrez, Cabero y Estrada (2017), Landa y Ramírez (2018), López y Norzagaray (2016), Ortega, Martínez y Nieto (2016). En los cuáles se validaron instrumentos para evaluar: la competencia digital, la satisfacción y percepción con respecto al modelo de aula invertida, las razones de los estudiantes para ingresar a la universidad y el aprendizaje creativo, respectivamente. 
Con relación al currículo en educación superior, algunas investigaciones tienen el objetivo de verificar su impacto en la calidad educativa. El estudio realizado por Almonacid et al. (2009), evaluó la pertinencia de un programa educativo desde la perspectiva de los graduados. Para ello construyeron dos modelos, el primero de ellos integró la variable de pertinencia individual y la pertinencia social de su formación; mientras que el segundo modelo se enfocó en identificar el nivel de competencias instrumentales y el nivel de competencias sistémicas. Para su validación se realizó un análisis exploratorio, confirmatorio y de fiabilidad, dando un resultado favorable para ambos modelos, ya que las variables seleccionadas como indicadores son pertinentes al constructo.

Asimismo, Lira-Valdivia (2014) desarrolló un estudio para evaluar el currículo donde diseñó un cuestionario de 248 ítems para valorar indicadores del proceso curricular, considerando dimensiones como: a) los principios curriculares, b) el perfil de ingreso y egreso, c) la estructura curricular, d) los elementos normativos institucionales, e) la autorregulación, f) la tutoría estudiantil, g) la infraestructura, y h) el seguimiento e idoneidad del currículo. Además, en esta investigación evaluativa, se efectuó la validez de contenido, los análisis para determinar la validez de constructo y la confiabilidad del instrumento, permitiendo respaldar la calidad y consistencia interna de dicho cuestionario.

Adicionalmente, González, Carabantes y Muñoz (2016) construyeron una escala con el propósito de conocer la perspectiva de los estudiantes sobre la calidad y cumplimiento de los programas de asignatura. En esta escala tipo Likert compuesta por 20 reactivos se consideran distintos elementos de los servicios educativos como los son la calidad del programa en general, de objetivos y contenidos, de la didáctica y evaluación; así como el cumplimiento de aspectos formales.

En este mismo sentido, Glaría, Carmona, Pérez y Parra (2016) realizaron un análisis factorial y de consistencia interna de una escala tipo Likert de 58 ítems, para la evaluación del currículum de programas universitarios de Fonoaudiología de una universidad tradicional de Concepción en Chile. Dicho instrumento, fue una adaptación de una escala elaborada por Schaufele (2011, citado por Glaría et al., 2016) para la evaluación de programas, la cual consta de ocho dimensiones: a) pertinencia del Currículum, b) metodología de enseñanza y evaluación, c) distribución del tiempo, d) objetivos de las asignaturas, e) objetivos de la carrera, f) distribución de actividades de enseñanza-aprendizaje, g) equipo docente y h) cumplimiento de objetivos. Los resultados del análisis factorial permitieron confirmar las ocho dimensiones del instrumento. Así mismo, se obtuvo la consistencia interna a través del valor de Alfa de Cronbach de las dimensiones, los cuales se ubicaron entre 0,74 y 0,86.

Al considerar las investigaciones anteriores, se puede identificar que, al llevar a cabo procesos de evaluación curricular, las dimensiones de los instrumentos coinciden en los siguientes aspectos: a) pertinencia o idoneidad, b) malla o estructura curricular, c) competencias docentes, d) estrategias de enseñanza y aprendizaje, e) infraestructura. Sin embargo, no se consideran el proceso desde los diferentes niveles de concreción curricular, ni la participación de los diversos actores del proceso; excepto, en el estudio de LiraValdivia (2014), que es una investigación más amplia, dónde se valoran los elementos normativos instituciones y los principios curriculares.

Debido a la necesidad de abordar los procesos del diseño curricular de una manera más holística, el enfoque socioformativo presenta una propuesta para desarrollar el currículo, considerando la formación de los diferentes actores del proceso educativo (directores, docentes, familias y estudiantes); con la finalidad de favorecer la formación integral a 
través de la comprensión y atención de problemas del contexto de manera colaborativa, así como la articulación de los diferentes saberes, con idoneidad y compromiso ético (Tobón, 2017).

Este enfoque presenta de manera integrada todos los elementos curriculares el contexto, los aspectos políticos, administrativos, financieros y educativos, a través socioformación; además, considera este proceso como permanente, transformador, continuo y de impacto en la atención de problema sociales (Tobón, 2017). Una de las principales características del enfoque, es que establece como aspecto fundamental el abordaje de los problemas del contexto en un momento determinado, como directriz del currículo; debido a que esto permite atender necesidades inmediatas, generando la reflexión y creatividad para la resolución de dicha problemática, con la finalidad de mejorar la calidad de vida, así como el desarrollo social y económico del contexto en el cual se desarrolla el proceso educativo (Prado, 2018; Tobón, 2017).

Una de las ventajas de efectuar una propuesta de diseño curricular por competencias bajo el enfoque socioformativo, es que permite atender cuatro retos principales: 1) la articulación con una visión global con la gestión curricular; 2) integrar el proceso como parte de la gestión de la calidad académica; 3) relacionarlo con la gestión de recursos, alianzas, gestión del talento humano y medición de resultados; y 4) planear las competencias bajo un proceso sencillo, a fin de facilitar que los docentes se impliquen y contribuyan en el proceso (Tobón, 2013).

Para desarrollar el diseño curricular bajo este enfoque, Tobón (2013) propone un modelo de Gestión Sistémica de la Formación por Competencias, integrado por doce fases que se desarrollan de manera sistémica y que son considerados como los ejes del currículo. La primera de ellas se enfoca en establecer el plan de acción a partir de las áreas de mejora del plan de estudios, para ello se requiere un equipo colaborativo que lidere el proceso. En la segunda fase se diseña el documento que integra el modelo educativo fundamentado bajo el enfoque socioformativo. En la tercera fase se realiza un estudio que considera el contexto interno y externo del entorno en el cual se desarrollará el currículo, estableciendo los retos como resultado de este proceso. Posterior a ello, se definen las competencias que se lograrán con el plan de estudios, así como el proceso que seguirá el estudiante para lograr su egreso. Durante la quinta fase se define el perfil que se requiere para el estudiante que ingresa al plan de estudios, así como las acciones de apoyo para reforzar sus competencias y el programa propedéutico a seguir. En la sexta fase se diseña la malla curricular donde se establecen los espacios de formación que cursará el estudiante (ver la figura 1).

La fase siete establece el reglamento de formación del estudiante, especificando el proceso de formación y evaluación de las competencias; continuando con la integración de los procesos de gestión académica que incluyen los aspectos docentes, de investigación, extensión y bienestar estudiantil. La novena fase formaliza la planeación y diseño de los espacios formativos, tales como proyectos.

En la décima fase se establecen acciones que permiten la gestión del talento humano, con el fin de contar con personal competente y que este se mantenga continuamente capacitado. Asimismo, se generan las políticas de gestión de recursos que atienden las acciones planteadas. Por último, es necesario definir las estrategias que favorecerán la formación integral del estudiante, contando con evidencias de este proceso de formación, como se muestra en la figura 1 (Tobón, 2013). 
Figura 1. Síntesis del modelo de Gestión Sistémica de la Formación por Competencias (Tobón, 2013).

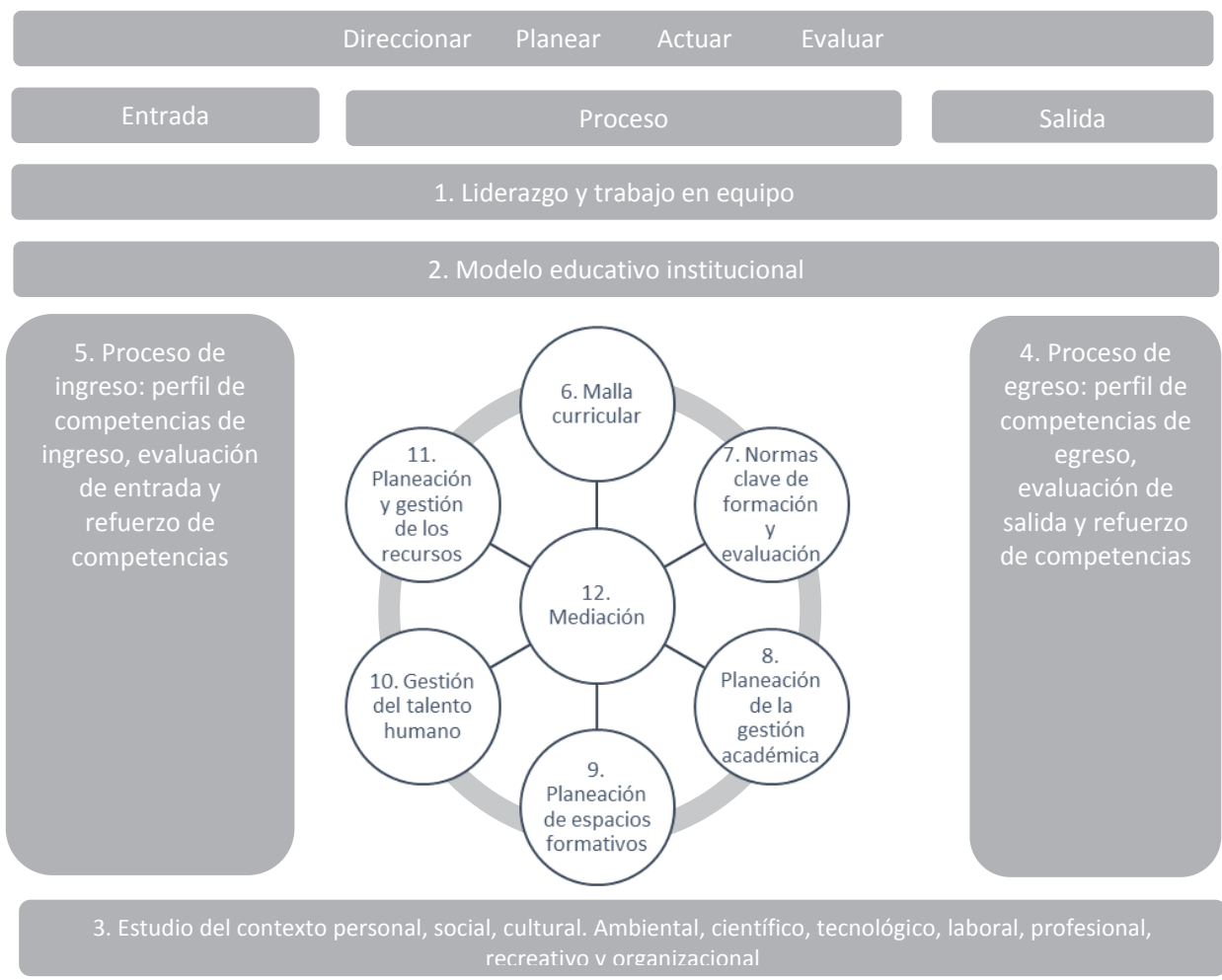

Otra de las características del enfoque, es que se deben de utilizar instrumentos más pertinentes para llevar a cabo la evaluación curricular, ya que se identifica la importancia de favorecer la actuación de las personas en los problemas del contexto. Debido a lo anterior, se propone una rúbrica analítica para facilitar la evaluación integral de un plan de estudios o del curriculum, en la cual se establezcan los indicadores de calidad para definir criterios que midan las evidencias y productos desarrollados durante el proceso de diseño curricular, así como sus niveles de desempeño posibles, acorde a dichos indicadores, con la finalidad de identificar el nivel de logro y las áreas de oportunidad (Hernández, Tobón y Guerrero, 2016; Tobón, 2014).

Dadas las condiciones que anteceden, se diseñó una rúbrica para evaluar la metodología empleada en el diseño curricular bajo el enfoque socioformativo (Tobón, 2013), considerando como criterios teóricos las 12 fases para su desarrollo: 1) planeación del liderazgo y equipo de trabajo, 2) planeación de la realización o mejoras del modelo educativo institucional, 3) estudio del contexto interno y externo, 4) planeación del perfil de egreso y proceso de egreso, 5) planeación del proceso de ingreso por competencias, 6) planeación de la malla curricular, 7) reglamento de formación y evaluación, 8) planeación de la gestión académica, 9) planeación de espacios formativos, 10) gestión del talento humano por competencias, 11) planeación y gestión de recursos; y, 12) mediación para la formación integral, el aprendizaje de las competencias y calidad de vida. 
Es importante mencionar que las propuestas establecen escalas tipo Likert para llevar a cabo el proceso de evaluación, las cuales suelen ofrecer buenos resultados debido a su bajo grado de ambigüedad, el tiempo que se utiliza para recabar la información y favorecer el propósito de la investigación. No obstante, este tipo de instrumento no permite resultados concluyentes debido al lenguaje utilizado, las escalas y las características de los individuos, ya que deben ser adaptadas de acuerdo al contexto (Cañadas y Sánchez, 1998; Kerlinger y Lee, 2008).

Por las consideraciones anteriores, en el presente estudio se decidió diseñar una rúbrica analítica, debido a que este tipo de instrumentos permite una evaluación integral del objeto de estudio a partir de niveles. En relación con este último, las rúbricas posibilitan una realimentación continua sobre lo que se espera de los indicadores y evidencias, valorados de manera cualitativa y cuantitativa; además, favorece la metacognición y mejora de la práctica educativa (Hernández et al., 2016).

Ante la situación planteada, el objetivo de este estudio fue establecer la validez de constructo y confiabilidad de una rúbrica para evaluar la aplicación de los 12 ejes esenciales de un currículo por competencias, bajo el enfoque socioformativo, en programas de licenciatura.

\section{MÉTODO}

Se realizó un estudio de validez de constructo de una rúbrica para autoevaluar la gestión de un currículo de licenciatura bajo el enfoque socioformativo, a partir de un análisis factorial exploratorio, con la finalidad de identificar si la interrelación de los ítems es suficiente y las variables comparten factores comunes (Pérez, Chacón y Moreno, 2000; Kerlinger y Lee, 2008; Lloret, Ferreres, Baeza y Tomás, 2014).

\subsection{PARTICIPANTES}

Para la realización de esta investigación, se aplicó el instrumento a 74 personas responsables de gestionar el currículo de programas educativos de licenciatura en 30 universidades de México y otros países de Latinoamérica: 63.5\% (47) México, 20.3\% (15) Chile, $8.1 \%$ (6) Colombia, 5.4\% (4) Guatemala, $1.4 \%$ (1) Perú y $1.4 \%$ y (1) Costa Rica. El modelo educativo de la mayoría de las universidades se basa en un curriculum bajo el enfoque por competencias $62 \%$ (46), otras trabajan bajo el enfoque humanista $25 \%$, (18) y solamente $13 \%$ (10) bajo el socioformativo (ver tabla 1).

Tabla 1. Modelo educativo utilizado en los programas de las universidades participantes

\begin{tabular}{|c|c|}
\hline Tipo de modelo educativo & Porcentaje \\
\hline Competencias & $62 \%$ \\
Socioformativo & $13 \%$ \\
Otros (humanista, CDIO, tradicional) & $25 \%$ \\
\hline
\end{tabular}

Fuente: Elaboración propia. 
En cuanto a las fechas de creación de los programas educativos y del último rediseño curricular, la mayoría de fue creado en: a) entre 1991 y 2010 (43.2\%) y b) de 1971 a 1990 (25.7\%); además, la gran mayoría de los programas educativos $(71.6 \%)$ fueron rediseñados entre el año 2015 y el 2019 (ver tabla 2).

Tabla 2. Fechas de creación y rediseño de los programas educativos

\begin{tabular}{|c|c|c|}
\hline Programa & Fechas de creación & Porcentajes \\
\hline \multirow{3}{*}{ Creación } & 1950 a 1970 & $8.1 \%$ \\
& 1971 a 1990 & $25.7 \%$ \\
& 1991 a 2010 & $43.2 \%$ \\
& 2011 a 2019 & $18.9 \%$ \\
& no contestó & $4.1 \%$ \\
\hline \multirow{3}{*}{ Último rediseño curricular } & $1999-2004$ & $2.7 \%$ \\
& $2005-2009$ & $2.7 \%$ \\
& $2010-2014$ & $18.9 \%$ \\
& $2015-2019$ & $71.6 \%$ \\
& no contestó & $4.1 \%$ \\
\hline
\end{tabular}

Fuente: elaboración propia.

\subsection{INSTRUMENTO}

Se diseñó una rúbrica para valorar la metodología utilizada en el diseño del currículo de licenciatura, bajo el enfoque socioformativo. La rúbrica analítica integró doce ítems, acordes a las fases de desarrollo curricular propuestas en el enfoque antes mencionado (ver tabla 3) y cinco niveles de desempeño: 0) nivel muy bajo, 1) nivel bajo, 2) nivel medio, 3) nivel medio alto, y 4) nivel muy alto. Cabe señalar que este instrumento fue sometido a una validez de contenido mediante un juicio de expertos, obteniendo valores mayores a 0.75 según el coeficiente de "V" de Aiken en cuanto a su pertinencia y redacción. Así mismo, se aplicó a un grupo piloto de 43 docentes y personal encargado del diseño curricular de universidades obteniendo una confiabilidad de 0.90 acorde al Alfa de Cronbach (Crespo, Mortis, Herrera y Manig, 2019). 
Tabla 3. Rúbrica para autoevaluar los ejes esenciales de un currículo bajo el enfoque socioformativo

\begin{tabular}{|c|c|}
\hline Fases & Ítem \\
\hline $\begin{array}{l}\text { Planeación del } \\
\text { liderazgo y equipo de } \\
\text { trabajo }\end{array}$ & $\begin{array}{l}\text { ¿En qué grado se tiene un equipo líder para gestionar el currículo, con } \\
\text { acciones concretas y periódicas que aseguren su pertinencia? }\end{array}$ \\
\hline $\begin{array}{l}\text { Planeación de la } \\
\text { realización o mejoras } \\
\text { del modelo educativo } \\
\text { institucional }\end{array}$ & $\begin{array}{l}\text { ¿En qué grado se propone un modelo educativo que responde a los retos } \\
\text { de formar para la sociedad del conocimiento, mediante el énfasis en la } \\
\text { resolución de problemas del entorno con pensamiento complejo? }\end{array}$ \\
\hline $\begin{array}{l}\text { Estudio del contexto } \\
\text { interno y externo }\end{array}$ & $\begin{array}{l}\text { ¿En qué grado el diseño curricular del programa tiene como base un } \\
\text { diagnóstico sistemático y formal de los retos y problemas del contexto tanto } \\
\text { de la actualidad como del futuro, considerando lo local y lo global, con el fin } \\
\text { de orientar la formación, la investigación y la vinculación con la sociedad? }\end{array}$ \\
\hline $\begin{array}{l}\text { Planeación del perfil } \\
\text { de egreso y proceso de } \\
\text { egreso }\end{array}$ & $\begin{array}{l}\text { ¿En qué grado se tiene un perfil de egreso basado en competencias que se } \\
\text { orienta a resolver los problemas y afrontar los retos identificados en el } \\
\text { estudio del contexto? }\end{array}$ \\
\hline $\begin{array}{l}\text { Planeación del proceso } \\
\text { de ingreso por } \\
\text { competencias }\end{array}$ & $\begin{array}{l}\text { ¿En qué grado se posee un perfil de ingreso en el cual se describen con } \\
\text { detalle las competencias básicas y genéricas mínimas que deben poseer } \\
\text { los estudiantes al inicio del programa para lograr con éxito el perfil de } \\
\text { egreso, tener continuidad en los estudios y prevenir la deserción? }\end{array}$ \\
\hline $\begin{array}{l}\text { Planeación de la malla } \\
\text { curricular }\end{array}$ & $\begin{array}{l}\text { ¿En qué grado la malla curricular del programa se orienta a la multi, inter } \\
\text { y transdisciplinariedad con espacios de formación articulados y en poca } \\
\text { cantidad por semestre, centrados en problemas? }\end{array}$ \\
\hline $\begin{array}{l}\text { Reglamento de } \\
\text { formación y } \\
\text { evaluación }\end{array}$ & $\begin{array}{l}\text { ¿En qué grado se tiene un sistema de evaluación y formación que enfatiza } \\
\text { en la evaluación continua y formativa centrada en el mejoramiento } \\
\text { continuo y el desarrollo del talento a través de productos de aplicación y } \\
\text { el empleo de instrumentos que sean comprendidos por los estudiantes } \\
\text { desde el inicio? }\end{array}$ \\
\hline $\begin{array}{l}\text { Planeación de la } \\
\text { gestión académica }\end{array}$ & $\begin{array}{l}\text { ¿En qué grado se tiene una política concreta de investigación en el } \\
\text { programa mediante líneas de investigación que articulan los espacios } \\
\text { formativos y el trabajo de los docentes y estudiantes, considerando los } \\
\text { problemas del contexto? }\end{array}$ \\
\hline $\begin{array}{l}\text { Gestión del talento } \\
\text { humano por } \\
\text { competencias }\end{array}$ & $\begin{array}{l}\text { ¿En qué grado se propone un cuerpo directivo, docente y administrativo } \\
\text { con la descripción de las competencias necesarias para poner en marcha } \\
\text { el currículo, junto con un proceso de formación continua y la evaluación } \\
\text { periódica del desempeño, que lleven a que el programa tenga impacto en } \\
\text { la formación de las personas de acuerdo con la sociedad del conocimiento? }\end{array}$ \\
\hline $\begin{array}{l}\text { Planeación de espacios } \\
\text { formativos }\end{array}$ & $\begin{array}{l}\text { ¿En qué grado se presenta el microcurrículo centrado en la resolución de } \\
\text { problemas del entorno, la evaluación por medio de productos e } \\
\text { instrumentos, y el desarrollo de competencias genéricas para la sociedad } \\
\text { del conocimiento, tales como el pensamiento crítico, la sustentabilidad } \\
\text { ambiental, la colaboración, el emprendimiento, el inglés, la comunicación } \\
\text { escrita, la gestión del conocimiento, etc? }\end{array}$ \\
\hline
\end{tabular}




\begin{tabular}{|l|l|}
\hline $\begin{array}{l}\text { Planeación y gestión } \\
\text { de recursos }\end{array}$ & $\begin{array}{l}\text { ¿En qué grado se proponen recursos que ayuden a formar para la sociedad } \\
\text { del conocimiento, como laboratorios o diversos ambientes de aprendizaje } \\
\text { que permitan resolver problemas del entorno o las tecnologías de la } \\
\text { información y la comunicación que posibiliten comunidades de } \\
\text { aprendizaje para el desarrollo social? }\end{array}$ \\
\hline $\begin{array}{l}\text { Mediación para la } \\
\text { formación integral, el } \\
\text { aprendizaje de las } \\
\text { competencias y la } \\
\text { calidad de vida }\end{array}$ & $\begin{array}{l}\text { ¿En qué grado se establecen lineamientos concretos para que las clases se } \\
\text { enfoquen en que los estudiantes aprendan a identificar, interpretar, } \\
\text { argumentar y resolver problemas del entorno, actuales y futuros, mediante } \\
\text { la colaboración, la co-creación de saberes, la aplicación de la } \\
\text { metacognición y el abordaje del proyecto ético de vida? }\end{array}$ \\
\hline
\end{tabular}

Fuente: elaboración propia.

\subsection{PROCEDIMIENTO}

Para realizar la validación de constructo del instrumento se llevaron a cabo las siguientes fases:

1. Análisis factorial exploratorio. Este análisis se desarrolló con la finalidad de observar cómo se agrupan los ítems de la escala y si esta agrupación guarda relación con los elementos teóricos revisados, en este caso, acorde a los ejes esenciales de un currículo bajo el enfoque socioformativo. Para esto se utilizó la extracción de componentes principales (Lloret et al., 2014), así mismo se consideró el valor propuesto por De Vellis (2012), quién indica que se debe contar con una carga factorial mayor a 0.30 como condición mínima del factor.

2. Análisis factorial confirmatorio. Esta técnica considera el análisis de la estructura de las varianzas, con la finalidad de verificar la consistencia del instrumento con la realidad, es decir, los factores esperados, los relacionados, así como aquello ítems que corresponden a cada factor (Lloret et al., 2014; Lévy y Varela, 2006). Para ello se decidió aplicar el método de estimación de máxima verosimilitud con la finalidad de confirmar la bondad empírica del modelo (Byrne, 2010; Cea, 2004).

3. Análisis de confiabilidad. Se llevó a cabo el análisis de confiabilidad de la rúbrica a partir del coeficiente de confiabilidad Alpha de Cronbach, el cual permite identificar la consistencia interna, precisión y fiabilidad de un instrumento (Soler y Soler, 2012; Kerlinger y Lee, 2008).

\section{RESULTADOS}

\subsection{ANÁLISIS FACTORIAL EXPLORATORIO}

En primer análisis realizado consistió en un análisis factorial exploratorio, mediante el método de factorización de ejes principales, así como los de rotación Oblimin. Los resultados mostraron que el valor del KMO es de 0.92 y un valor de $\mathrm{X}^{2}=645.02(\mathrm{P}<.000)$ acorde a la esfericidad de Barlett, los cuales se ubican en niveles pertinentes para dichas pruebas. Además, los resultados mostraron que existe una solución unifactorial, ya que se 
identificó un $63.3 \%$ en la varianza de los puntajes, es decir, el instrumento permite valorar la gestión de un currículo por competencias bajo el enfoque socioformativo, en programas de licenciatura, mediante la aplicación de sus ejes esenciales (Ver tabla 4).

Tabla 4. Resultados del Análisis Factorial Exploratorio de la Rúbrica para evaluar la gestión del currículo por competencias bajo el enfoque socioformativo de licenciatura

\begin{tabular}{|c|c|c|c|c|}
\hline \multirow{2}{*}{ Ítem } & \multicolumn{4}{|c|}{ Carga Factorial } \\
\cline { 2 - 5 } & $M$ & $D E$ & $\mathrm{~F} 1$ & $\mathrm{~h}^{2}$ \\
\hline Ítem 1. & 2.55 & 1.07 & $\mathbf{. 8 7}$ & $\mathbf{. 5 8}$ \\
\hline Ítem 2. & 2.82 & 1.07 & $\mathbf{. 8 4}$ & $\mathbf{. 6 5}$ \\
\hline Ítem 3. & 2.74 & 1.05 & $\mathbf{. 8 2}$ & $\mathbf{. 6 7}$ \\
\hline Ítem 4. & 3.19 & .95 & $\mathbf{. 8 1}$ & $\mathbf{. 5 2}$ \\
\hline Ítem 5. & 2.46 & 1.21 & $\mathbf{. 8 1}$ & $\mathbf{. 6 5}$ \\
\hline Ítem 6. & 2.51 & 1.01 & $\mathbf{. 8 0}$ & $\mathbf{. 5 9}$ \\
\hline Ítem 7. & 2.31 & 1.10 & $\mathbf{. 7 8}$ & $\mathbf{. 5 7}$ \\
\hline Ítem 8. & 2.43 & 1.13 & $\mathbf{. 7 7}$ & $\mathbf{. 6 6}$ \\
\hline Ítem 9. & 2.54 & 1.06 & $\mathbf{. 7 6}$ & $\mathbf{. 7 5}$ \\
\hline Ítem 10. & 2.56 & 1.01 & .76 & .58 \\
\hline Ítem 11. & 2.60 & 1.12 & .76 & .60 \\
\hline Ítem 12. & 2.58 & 1.00 & .72 & .70 \\
\hline
\end{tabular}

Fuente: elaboración propia.

\subsection{ANÁLISIS FACTORIAL CONFIRMATORIO}

Para la realización del análisis factorial confirmatorio se empleó el método de estimación de máxima verosimilitud para determinar la bondad de ajuste empírica del modelo (ver figura 2), es decir, permitió comprobar que la rúbrica considera los aspectos teóricos de la metodología establecida para evaluar los ejes esenciales del currículo o gestión sistémica de la formación por competencias, para el desarrollo de programas de licenciatura bajo el enfoque socioformativo (Byrne, 2010; Cea, 2004). 
Figura 2. Solución unifactorial para medir los ejes esenciales de un currículo bajo el enfoque socioformativo.

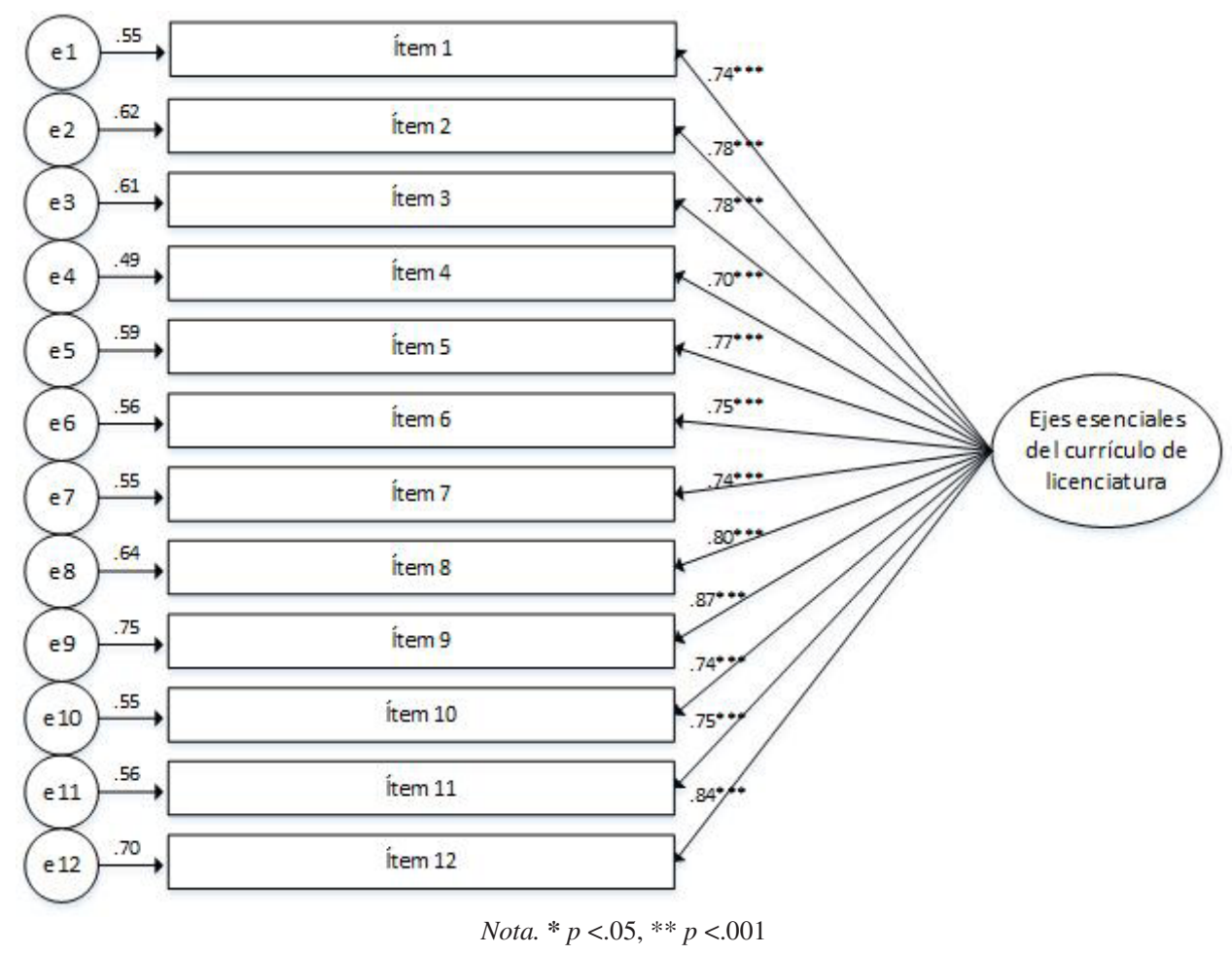

Fuente: Elaboración propia.

Además, se consideraron los índices de ajuste, los cuales permiten confirmar lo establecido de manera empírica en el modelo (Blunch, 2013; Byrne, 2010; Cea, 2004). Es decir, se confirma que el modelo es unidimensional, ya que todos los índices de ajuste son satisfactorios, para el desarrollo de las fases curriculares desde la socioformación (ver tabla 5).

Tabla 5. Índices de Ajuste del Modelo para las Evaluar los ejes esenciales del currículo bajo el enfoque socioformativo

\begin{tabular}{|c|c|c|c|c|c|c|}
\hline Modelo & $X^{2}$ & $P$ & $X^{2} / g l$ & $C F I$ & $R M S E A$ & $G F I$ \\
\hline Unifactorial & 80.82 & $.008^{* *}$ & 1.525 & .95 & .052 & .85 \\
\hline
\end{tabular}

Nota. $C F I=I ́$ dice de ajuste comparativo; $R M S E A=$ Error de la raíz cuadrada media de aproximación; $G F I=$ Índice de bondad de ajuste * $p<.05$. ** $p<.01$. *** $p<.001$. 


\subsection{CONFIABILIDAD}

Los resultados obtenidos mediante el análisis de confiabilidad arrojaron un valor de 0.94 para el coeficiente de Alfa de Cronbach, es decir, indican que de los doce ítems que integran la rúbrica para evaluar los ejes esenciales del currículo bajo el enfoque socioformativo, cuenta con una buena consistencia interna. Este valor es superior al obtenido en un estudio anterior, con un grupo piloto de 43 participantes en el cual se obtuvo un valor de Alfa de Cronbach de 0.90 (Crespo et al., 2019).

\section{DISCUSIÓN Y CONCLUSIONES}

La rúbrica fue elaborada para medir la gestión de un currículum por competencias bajo el enfoque socioformativo en programas educativos de licenciatura, es decir, el grado de aplicación de los 12 ejes esenciales para la gestión sistémica de la formación en esta perspectiva curricular. Se logró el objetivo de este estudio, que fue analizar las propiedades métricas de dicho instrumento, es decir, la validez de constructo y su consistencia interna.

A partir de los análisis realizados, se puede establecer que la rúbrica es adecuada para evaluar los ejes esenciales de un currículo de licenciatura bajo el enfoque socioformativo, lo cual se determinó al realizar la validez de constructo por medio de un análisis factorial exploratorio de ejes principales, obteniendo valores mayores a 0.30 en la carga factorial. Lo anterior también se puede identificar en los estudios de validez de instrumentos realizados por Tobón et al. (2018), López y Norzagaray (2016), Lira-Valdivia (2014), González et al. (2016), así como de Landa y Ramírez (2018).

Así mismo, se realizó otro análisis para confirmar la validez de constructo de dicha rúbrica, se identifica que este es pertinente, ya que los valores de KMO obtenidos fueron mayores a 0.30 . Significa entonces, que la rúbrica permite medir el factor relacionado a las fases del desarrollo curricular de un programa de licenciatura. Lo anterior coincide con resultados obtenidos por Almonacid et al. (2009), quienes realizaron un análisis factorial confirmatorio para dos modelos que permiten evaluar la pertinencia de un programa académico, con relación a las necesidades del mercado laboral, las competencias, competencia profesional y su pertinencia social.

De igual forma, se considera que la consistencia interna de la rúbrica es pertinente, ya que cuenta con un Alfa de Cronbach de .94, los cuál indica que es bastante aceptable, debido a que, este coeficiente debe ser mayor a 0.80 para este tipo de instrumentos (Kerlinger y Lee, 2008). Lo anterior, es coincidente con el valor obtenido en la validación de los estudios realizados por Agreda et al. (2016), Lira-Valdivia (2014), Glaría et al. (2016), Gutiérrez et al. (2017); así como de Ortega et al. (2016).

Cabe mencionar que actualmente existen instrumentos que permiten medir el diseño curricular bajo el enfoque por competencias, los cuales toman en cuenta algunos de los elementos propios de este proceso. Sin embargo, no se cuenta con información que confirme que se hayan diseñado instrumentos para medir el diseño curricular por competencias bajo la socioformación, por lo que la rúbrica analítica propuesta en es este estudio permitirá identificar el nivel de aplicación de los ejes esenciales de un curriculum por competencias bajo dicho enfoque, en programas educativos de licenciatura, así como 
a los requerimientos de la sociedad del conocimiento, y sus resultados en la formación de los profesionistas en las universidades del país y de Latinoamérica.

Las instituciones de educación superior deben enfocarse en la formación integral, con la finalidad de favorecer no solo los aspectos técnicos, sino los valores, la reflexión y el análisis crítico para favorecer tanto el componente humano como profesional (Paredes e Inciarte, 2013; Vidal, Salas, Fernández y García, 2016). Debido a lo anterior, es importante recalcar que el reto que tienen las universidades no es sencillo, ya que cuando se desarrollan actualizaciones curriculares, se tiene como objetivo mantener la pertinencia e incrementar la calidad de los programas educativos, a fin de atender y resolver los problemas que se viven dentro de la sociedad, a través del desarrollo profesional y el crecimiento personal del ser humano.

\section{REFERENCIAS BIBLIOGRÁFICAS}

Agreda, M., Hinojo, M., y Sola, J. (2016). Diseño y validación de un instrumento para evaluar la competencia digital de los docentes en la educación superior española. Píxel-Bit. Revista de medios y educación, 49, 39-56. Doi: http://dx.doi.org/10.12795/pixelbit.2016.i49.03

Almonacid, P., Montes, I. C. y Vásquez, J. J. (2009). Un análisis factorial para evaluar la pertinencia de un programa académico desde la perspectiva de los graduados: un estudio de caso. Revista ecos de economía, 13(29). Última visita 10 de agosto de 2019. Recuperado desde https://dialnet. unirioja.es/ servlet/ articulo?codigo $=4024697$

Blunch, N. J. (2013). Introduction to structural equation modeling using IBM SPSS statistics and $A M O S\left(2^{\text {nd }}\right.$ ed.). London: SAGE.

Byrne, B. (2010). Structural equation modeling with AMOS (2 ${ }^{\text {nd }}$ ed.). New York: Routledge Taylor $\&$ Francis Group.

Cañadas, I., y Sánchez, B. (1998). Categorías de respuesta en escalas tipo Likert. Psicothema, 10(3), 623-631. Última visita 09 de agosto de 2019. Recuperado desde http://www.psicothema.com/ pdf/191.pdf

Castro, F., Lira, H., y Castañeda, M. (2017). Estudio evaluativo del diseño e implementación curricular de la formación pedagógica en carreras de educación. Revista actualidades investigativas en educación, 17(2), 1-23. DOI: 10.15517/aie.v17i2.28675

Cea, M. (2004). Análisis multivariable. Teoría y práctica en la investigación social. Madrid: Síntesis.

Cevallos, D. (2014). La calidad educativa en la realidad universitaria peruana frente al contexto Latinoamericano. Revista de la universidad católica Santo Toribio de Mogrovejo, 7(1), 3-8. Última visita 09 de agosto de 2019. Recuperado desde http://repositorio.usat.edu.pe/handle/ usat $/ 104$

Crespo, A., Mortis, S., Herrera, S. y Manig, A. (2019). Diseño de una rúbrica para autoevaluar los ejes esenciales del currículo de licenciatura desde la socioformación. Revista Digital Atenas, 3(47), 142-160. Última visita 10 de octubre de 2019. Recuperado desde https://atenas.reduniv. edu.cu/index.php/atenas/ article/view/494/772

Cruz, L., Lemus, D., Ramos, M. y Pujols, A. (2017). Valoración del diseño curricular del programa de la asignatura Quiropodología. Revista de Ciencias Médicas de Pinar del Río, 21(5), 716724. Última visita 09 de octubre de 2019. Recuperado desde http://scielo.sld.cu/pdf/rpr/v21n5/ rpr14517.pdf

De Vellis, R. (2012). Scale development. Theory and applications. New York: Sage.

Glaría, R., Carmona, L., Pérez, C. y Parra, P. (2016). Estructura factorial y consistencia interna de la escala de evaluación del currículum de programas universitarios en estudiantes de fonoaudiología de Chile. Revista Iberoamericana de Diagnóstico y Evaluación - e Avaliação 
Psicológica. RIDE, 1(41), 80-89. Última visita 10 de agosto de 2019. Recuperado desde https:// www.aidep.org/sites/default/files/articles/R41/Art7.pdf

González, M. I., Carabantes, E. y Muñoz, N. E. (2016). Construcción y validación de la escala de apreciación de la calidad del programa de asignatura. Propuesta para el estudio de la calidad de la docencia. Revista de Formación Universitaria, 9(1), 77-90. Doi: http://dx.doi.org/10.4067/ S0718-50062016000100009

Gutiérrez, J. J., Cabero, J. y Estrada L. I. (2017). Diseño y validación de un instrumento de evaluación de la competencia digital del estudiante universitario. Revista Espacios, 38(10). Última visita 10 de agosto de 2019. Recuperado desde http://www.revistaespacios.com/a17v38n10/17381018. html

Hernández, J. S., Tobón, S. y Guerrero, G. (2016). Hacia una evaluación integral del desempeño: las rúbricas socioformativas. RA XIMHAI, 12(6), 359-376. Última visita 12 de agosto de 2019. Recuperado desde http://www.redalyc.org/pdf/461/46148194025.pdf

Icarte, G. A. y Labate, H. A. (2016). Metodología para la revisión y actualización de un diseño curricular de una carrera universitaria incorporando conceptos de aprendizaje basado en competencias. Revista de Formación universitaria, 9(2). Doi: http://dx.doi.org/10.4067/ S071850062016000200002.

Kerlinger, F. y Lee, H. (2008). Investigación del comportamiento. Métodos de investigación en ciencias sociales. México: McGrawHill.

Landa, M. R. y Ramírez, M. Y. (2018). Diseño de un cuestionario de satisfacción de estudiantes para un curso de nivel profesional bajo el modelo de aprendizaje invertido. Páginas de educación, 11(2), 153 -175. Doi: https://doi.org/10.22235/pe.v11i2.1632

Lévy, J. y Varela, J. (2006). Modelización con estructuras de covarianzas en ciencias sociales. España: Netbiblo.

Lira-Valdivia, R. I. (2014). Validación del constructo, contenido y confiabilidad de indicadores de proceso para la evaluación de la dimensión curricular en la educación superior: el caso del Instituto Tecnológico de Costa Rica. Revista Tecnología en marcha, 2(2), 117-136. Doi: https:// doi.org/10.18845/tm.v27i2.1932

López, D. y Norzagaray, C. C. (2016). Construcción y validación de un instrumento para evaluar factores disposicionales asociados a la elección de carrera. Enseñanza e investigación en psicología, 21(2), 120-130. Última visita 12 de agosto de 2019. Recuperado desde http://www. redalyc.org/ articulo.oa?id= 29248181002

Lloret, S., Ferreres, A., Baeza, A. \& Tomás, I. (2014). Exploratory Item Factor Analysis: A practical guide revised and up-dated. Anales De Psicología/Annals of Psychology, 30(3), 1151-1169. Doi: http://dx.doi.org/10.6018/analesps.30.3.199361

Martínez, J., Tobón, S. y Romero, A. (2017). Problemáticas relacionadas con la acreditación de la calidad de la educación superior en América Latina. Revista de innovación educativa, 17(73), 79-96. Última visita 12 de agosto de 2019. Recuperado desde http://www.scielo.org.mx/scielo. php?pid=S1665-26732017000100079\&script=sci_arttext\&tlng=pt

Ortega, M., Martínez, J. y Nieto, J. (2016). Validez de instrumento para medir el aprendizaje creativo. Revista Comunicaciones en Estadística, 9(2), 239-254. Doi: https://doi.org/10.15332/s20273355.2016.0002.04

Paredes, Í. e Inciarte, A. (2013). Enfoque por competencias. Hacia la integridad y el desempeño profesional con sentido social y crítico. Revista Omnia, 19(2), 125-138. Recuperado desde http://www.redalyc.org/articulo.oa?id=73728678010

Pérez, J., Chacón, S. y Moreno R. (2000). Validez de constructo: el uso de análisis factorial exploratorioconfirmatorio para obtener evidencias de validez. Psicothema, 12(Supl. 2), 442-446. Última visita 10 de octubre de 2019. Recuperado desde https://idus.us.es/xmlui/handle/11441/42748

Prado, R. (2018). La socioformación: un enfoque de cambio educativo. Revista Iberoamericana De Educación, 76(1), 57-82. Doi: https://doi.org/10.35362/rie7612955 
Soler, S. F. y Soler, L. (2012). Usos del coeficiente alfa de Cronbach en el análisis de instrumentos escritos. Revista Medica Electrónica, 34(1). Recuperado desde: http://scielo.sld.cu/scielo. php?script=sci_arttext\&pid=S1684-18242012000100001

Tobón, S. (2013). Formación integral y competencias: pensamiento complejo, currículo, didáctica y evaluación (4ta. Edición). Bogotá: Ecoe ediciones.

(2014). Rúbricas socioformativas. México: CIFE

(2017). Ejes esenciales de la sociedad del conocimiento y la socioformación. Mount Dora, Florida: Kresearch. DOI: 10.24944/isbn.978-1-945721-18-2

Tobón, S., Guzmán, C., Hernández, J. y Cardona, S. (2015). Sociedad del conocimiento: estudio documental desde una perspectiva humanista y compleja. Revista Paradigma, 36(2), 7-36. Última visita 09 de agosto de 2019. Recuperado desde http://ve.scielo.org/pdf/pdg/v36n2/art02.pdf

Tobón, S., Pimienta, J. H., Herrera, S. R., Juárez, L. G. y Hernández, J. S. (2018). Validez y confiabilidad de una rúbrica para evaluar las prácticas pedagógicas en docentes de educación media (SOCME-10). Revista Espacios, 39(53). Última visita 11 de octubre de 2019. Recuperado desde http://www.revistaespacios.com/cited2017/cited2017-30.pdf

Vidal, M.J., Salas, R. S., Fernández, B. y García, A. L. (2016). Educación basada en competencias. Revista Cubana de Educación Media Superior, 30(1). Última visita 10 de agosto de 2019. Recuperado desde https://www.medigraphic.com/pdfs/educacion/cem-2016/cem161r.pdf 
